\title{
CONSTRUCTION OF SAMPLING AND INTERPOLATING SEQUENCES FOR MULTI-BAND SIGNALS. THE TWO-BAND CASE
}

\author{
SERgei AVDONIN*, AnNA BULANOVA **, William MORAN *** \\ * Department of Mathematics and Statistics, University of Alaska Fairbanks \\ Fairbanks, AK 99775-6660, USA \\ e-mail: ffsaa@uaf.edu \\ ** Department of Mathematics and Statistics, University of Alaska Fairbanks \\ Fairbanks, AK 99775-6660, USA \\ e-mail: ftasb1@uaf.edu \\ *** Department of Electrical and Electronic Engineering, University of Melbourne \\ Melbourne Victoria 3010, Australia \\ e-mail: bmor@ee.mu.oz.au
}

\begin{abstract}
Recently several papers have related the production of sampling and interpolating sequences for multi-band signals to the solution of certain kinds of Wiener-Hopf equations. Our approach is based on connections between exponential Riesz bases and the controllability of distributed parameter systems. For the case of two-band signals we derive an operator whose invertibility is equivalent to the existence of a sampling and interpolating sequence, and prove the invertibility of this operator.
\end{abstract}

Keywords: sampling and interpolation, multi-band signals, Riesz bases, families of exponentials, Wiener-Hopf equations, control, observation

\section{Introduction}

Let $E$ be a finite union of disjoint intervals:

$$
\begin{aligned}
E & =\bigcup_{j=1}^{N} I_{j}, \quad I_{j}=\left[a_{j}, b_{j}\right], \\
0 & =a_{1}<b_{1}<a_{2}<b_{2}<\ldots<a_{N}<b_{N} .
\end{aligned}
$$

Several papers (Avdonin and Moran, 1999; Bezuglaya and Katsnelson, 1993; Katsnelson, 1996; Lyubarskii and Seip, 1997; Lyubarskii and Spitkovsky, 1996; Moran and Avdonin, 1999; Seip, 1995) have recently appeared that discuss Riesz bases of exponentials in $L^{2}(E)$. All of them emphasize the importance of this problem in communication theory: if $\left\{e^{i \lambda_{k} t}\right\}$ forms a Riesz basis in $L^{2}(E)$ then $\Lambda=\left\{\lambda_{k}\right\}$ is a sampling and interpolating set for the corresponding multi-band signals. In other words, the interpolation problem

$$
s\left(\lambda_{k}\right)=\alpha_{k}, \quad \lambda_{k} \in \Lambda, \quad s \in L_{E}^{2},
$$

has a unique solution for each $\left\{\alpha_{k}\right\} \in l^{2}$. Here $L_{E}^{2}$ is the space of entire functions of the form

$$
s(\lambda)=\int_{E} e^{i \lambda t} \phi(t) \mathrm{d} t, \quad \phi \in L^{2}(E),
$$

endowed with the $L^{2}(\mathbb{R})$ norm. The equivalence of these two problems is well known; it follows from standard duality arguments (see, e.g., (Hruščev et al., 1981; Lyubarskii and Seip, 1997)).

It is interesting to note that the papers (Avdonin and Moran, 1999; Katsnelson, 1996; Lyubarskii and Spitkovsky, 1996) have related the production of Riesz bases to the invertibility of certain convolution integral operators. The method of (Katsnelson, 1996) is based on the mean periodic continuation of a function with respect to a finite measure. The convolution operator in (Lyubarskii and Spitkovsky, 1996) is constructed on a union of intervals connected with the entire function generating the set $\Lambda$.

Another approach to the problem was proposed in the paper (Avdonin and Moran, 1999). It is based on con- 
nections between the controllability of a dynamical system described by a linear PDE and the Riesz basis property of a corresponding exponential family. These connections are well known and widely exploited in control theory; see, e.g., the excellent survey paper (Russell, 1978) and the book (Avdonin and Ivanov, 1995). The problem of constructing an exponential basis on several intervals gives rise to a new type of control problem with boundary control supported on these time intervals.

More precisely, (Avdonin and Moran, 1999) introduced an auxiliary dynamical system described by the string equation with boundary control $u$ :

$$
\begin{aligned}
\rho^{2}(x) y_{t t}(x, t) & =y_{x x}(x, t), \\
y(0, t) & =u(t), \\
y_{x}(l, t) & =0
\end{aligned}
$$

where $\rho(x)$ is a positive function on $[0, l]$ which will be determined later. Usually, in control theory, the function $u$ is taken from $L^{2}(0, T)$ for some positive $T$, but for our purposes we take $u$ from $L_{\text {loc }}^{2}(\mathbb{R})$ with support restricted to $E$ and consider the initial conditions

$$
y\left(x, a_{1}\right)=y_{0}(x), y_{t}\left(x, a_{1}\right)=y_{1}(x) .
$$

The eigen-frequencies $\lambda_{n}, n \in \mathbb{N}$, of this system can be found from the boundary value problem

$\phi^{\prime \prime}(x)+\lambda^{2} \rho^{2}(x) \phi(x)=0,0<x<l, \phi(0)=\phi^{\prime}(l)=0$.

The system (1) is called exactly controllable if for any initial conditions $\left(y_{0}, y_{1}\right) \in L^{2}(0, l) \times H^{-1}(0, l)$ there is a unique control $u \in L^{2}(E)$ which brings the system to the origin at $t=b_{N}$ :

$$
y\left(\cdot, b_{N}\right)=y_{t}\left(\cdot, b_{N}\right)=0 .
$$

The following statement plays a key role in this approach to the construction of sampling and interpolating sequences.

Theorem 1. (Avdonin and Moran, 1999) The system (1) is exactly controllable if and only if the family $\left\{e^{ \pm i \lambda_{n} t}\right\}$ forms a Riesz basis in $L^{2}(E)$.

In other words, the exact controllability of (1) is equivalent to the fact that $\Lambda=\left\{ \pm \lambda_{n}\right\}$ is a sampling and interpolating sequence for $L_{E}^{2}$. Note that all of $\lambda_{n}^{2}$, i.e., the eigenvalues of the boundary value problem (3) are positive and we may therefore choose $\lambda_{n}$ to be positive.

Our problem then becomes that of constructing the function $\rho(x)$ in such a way that the system (1) is exactly controllable. If the set $E$ consists only of the interval $[a, b]$ and the control $u$ acts from $t=a$ to $t=b$, then, as is well known (see, e.g., (Avdonin and Ivanov, 1995; Russell, 1978)), the system (1) is exactly controllable if and only if the length of the interval is equal to two optical lengths of the string:

$$
b-a=2 \int_{0}^{l} \rho(x) \mathrm{d} x .
$$

Choosing $\rho=$ const (homogeneous string), we obtain the uniform sampling and interpolating sequence for $L_{[a, b]}^{2}$ :

$$
\Lambda= \pm \frac{2 \pi}{b-a}\left(n-\frac{1}{2}\right), \quad n \in \mathbb{N}
$$

Taking $\rho$ as a smooth (from $C^{2}[0,1]$ ) non-constant function, we obtain a non-uniform sampling and interpolating sequence asymptotically close to (4).

In the multi-band case, we cannot (in a general situation) produce a sampling and interpolating sequence taking $\rho$ as a constant or smooth function from $C^{2}[0,1]$. This fact can be understood by taking into account a necessary "geometric" condition for the controllability of the system (1): if the system (1) is exactly controllable, then for every $x_{0} \in[0, l]$ both characteristics starting at the point $x=x_{0}, t=0$ and lying in the strip $[0, l] \times\{t \geq 0\}$ of the $(x, t)$-plane have a non-empty intersection with $\{x=0\} \times E$. We suppose that the characteristics "reflect" from the boundaries subjecting to geometric optics laws.

For example, if $E=[0,1] \cup[2,3]$, none of the smooth functions $\rho$ satisfies the "geometric" condition. Using Theorem 1 it can also be proved that uniform sampling and interpolation of multi-band signals is possible only when very special relations exist between the lengths of intervals and gaps between them. More precisely, the special case is when $E$ is an explosion of an interval (Higgins, 1996, Sec. 13.1)

To satisfy the "geometric" controllability condition in the multi-band case, we should consider piecewise smooth functions $\rho$. More exactly, we take points $0=$ $x_{0}<x_{1}<\cdots<x_{N}=l$ and a piecewise constant function $\rho(x)$ such that

$$
\begin{gathered}
\rho(x)=\rho_{j}, \text { for } x_{j-1}<x<x_{j} ; 0<\rho_{j}<\infty, \\
\rho_{j} \neq \rho_{j+1},
\end{gathered}
$$

$$
\rho_{j}\left(x_{j}-x_{j-1}\right)=\left(b_{j}-a_{j}\right) / 2, j=1,2, \ldots, N .
$$

Due to the condition $\rho_{j} \neq \rho_{j+1}$ there are additional reflections of the waves from the boundaries $x=x_{j}$ of the "layers" which improve the controllability of the system (1). The analysis of the obtained control problem leads us to the following conjecture:

Conjecture 1. Let E be a multi-band set described above. Then, for all functions $\rho(x)$ satisfying (5), (6), the system (1) is exactly controllable. 
This conjecture was confirmed in some particular cases in (Avdonin and Moran, 1999), and we are working on its complete proof using PDE techniques.

Conjecture 1 implies that the exponential family $\left\{e^{ \pm i \lambda_{n} t}\right\}_{n \in \mathbb{N}}$ forms a Riesz basis in $L^{2}(E)$, where $\lambda_{n}^{2}$ are the eigenvalues of the boundary value problem (3) and $\rho(x)$ satisfies the conditions (5) and (6). It is important for applications that the sampling and interpolating set $\left\{ \pm \lambda_{n}\right\}$ is real.

The boundary-value problem (3), (5), (6) represents an important example of an eigenvalue problem whose spectrum generates a sampling and interpolation sequence for a multi-band signal.

In (Avdonin and Moran, 1999), the sampling and interpolation problem is reduced to the solution of difference equations. Specifically, they are Wiener-Hopf equations of a special form. The solution of the problem (1), (2) with $\rho(x)$ satisfying the conditions (5) and (6) can be written in an explicit, although rather complicated form. The analysis of that formula leads to invertibility problems for operators connected with difference equations. While this method appears to extend to handle arbitrary finite unions of intervals, we illustrate it in the case of two intervals.

Only a few results concerning sampling and interpolating sequences for the case when the set $E$ is a union of two intervals are known. (Kohlenberg, 1953) constructed a sampling and interpolating sequence for signals whose spectrum is restricted to the union of two intervals of the same length (band-pass signals). The later great impact to this field was due to Dodson and Silva (1989), Beaty and Dodson $(1989 ; 1993)$ and Bezuglaya and Katsnelson (1993). In these papers the lengths of the intervals and the gaps were supposed to have a special structure such as the commensurability of the lengths of the intervals and the gaps. (Lyubarskii and Seip, 1997) remark that the method of (Kohlenberg, 1953) can be extended to the case when the intervals comprising $E$ have commensurable lengths. The results of Seip (1995) are free of arithmetic restrictions on the lengths of the intervals comprising the set $E$; in particular, starting from the " $1 / 4$ in the mean" theorem (Avdonin, 1979), he gives a construction of at least one real sampling and interpolating sequence for an arbitrary $E$ consisting of two intervals.

The main result. The present paper is devoted to the investigation of the convolution operator proposed in (Avdonin and Moran, 1999) in the case when $E$ is the union of two arbitrary intervals. We prove that this operator is invertible if a parameter $\mu=\left(\rho_{2}-\rho_{1}\right) /\left(\rho_{2}+\rho_{1}\right)$ is small enough. The latter proves the existence of infinitely many real sampling and interpolating sequences for signals with the spectrum supported on two arbitrary intervals. We also give an algorithm for the construction of such sequences.

\section{Operators $W, V$ and $K$}

Let

$$
\begin{gathered}
E=I_{1} \cup I_{2}, \\
I_{j}=\left[a_{j}, b_{j}\right],\left|I_{j}\right|:=b_{j}-a_{j}=\alpha_{j}, \quad j=1,2, \\
\alpha_{1}+\alpha_{2}=\alpha, \quad a_{2}-b_{1}=\alpha^{\prime} .
\end{gathered}
$$

Note that, without loss of generality, we can assume that $\alpha_{1}$ is less than or equal to $\alpha_{2}$.

In (Avdonin and Moran, 1999), it was proved that the problem of constructing a sampling and interpolating sequence for $L_{E}^{2}$ can be reduced to the study of the invertibility of the operator

$$
W: L^{2}\left(0, \alpha_{1}\right) \mapsto L^{2}\left(\alpha^{\prime}, \alpha^{\prime}+\alpha_{1}\right)
$$

defined by

$$
\begin{aligned}
(W f)(t)= & \chi_{\left[\alpha^{\prime}, \alpha^{\prime}+\alpha_{1}\right]}(t) \\
& \times \sum_{r=0}^{\infty} \sum_{k=0}^{\infty} \sum_{q=0}^{k} A(r, k, q) f(t-w(r, k, q)),(9)
\end{aligned}
$$

where $w(r, k, q)=\alpha r+\alpha_{1}(k-q)+\alpha_{2} q$ and

$$
A(r, k, q)=(-1)^{r+k} \mu^{k} \frac{(r+k) !}{r ! q !(k-q) !}
$$

for $\mu \in(-1,0) \cup(0,1)$. Here

$$
\mu=\frac{\rho_{2}-\rho_{1}}{\rho_{2}+\rho_{1}},
$$

where $\rho_{1}$ and $\rho_{2}$ are the values of a piecewise constant density function of an associated string equation (1) satisfying the controllability conditions (5) and (6).

Once we find a parameter $\mu$ for which the operator $W$ is invertible, a sampling and interpolating sequence for signals with the spectrum supported on $E$ can be found using the following scheme:

\section{Algorithm 1.}

(a) Pick any two different values $\rho_{1}>0, \rho_{2}>0$ such that $\mu=\left(\rho_{2}-\rho_{1}\right) /\left(\rho_{2}+\rho_{1}\right)$.

(b) Find the numbers $l$ and $x_{1}$ from the equations

$$
\begin{aligned}
\rho_{1} x_{1} & =\frac{\alpha_{1}}{2}, \\
\rho_{2}\left(l-x_{1}\right) & =\frac{\alpha_{2}}{2} .
\end{aligned}
$$

(c) Define the density function

$$
\rho(x)=\left\{\begin{array}{lll}
\rho_{1} & \text { when } & 0<x<x_{1}, \\
\rho_{2} & \text { when } & x_{1}<x<l .
\end{array}\right.
$$


(d) Find the eigenvalues $\lambda_{n}^{2}$ of the boundary value system (3) with the function $\rho(x)$ and the number $l$ found in Steps (b) and (c). The sequence $\Lambda=\left\{ \pm \lambda_{n}\right\}$ is a sampling and interpolating sequence for $L_{E}^{2}$.

In the formula (9) and in what follows it is convenient to assume that $f$ is defined on the real axis with support in $\left[0, \alpha_{1}\right]$. One can see that in this case for each $t \in\left[\alpha^{\prime}, \alpha^{\prime}+\right.$ $\alpha_{1}$ ] the number of terms in the sum is finite, since only the terms with $t-w(r, k, q) \in\left[0, \alpha_{1}\right]$ are not equal to zero.

Our goal is to reduce the problem of the invertibility of the operator $W$ to a problem of the invertibility of a simpler operator. We are going to break the sum corresponding to the operator $W$ into two sums, $W=U+\widetilde{U}$, so that the operator $U$ is invertible and its invertibility implies that of $W$. We show that it is possible to make $U$ contain no more than four terms. The invertibility of the operator $U$ is proven in Theorems 7 and 8 .

Theorem 2. For any $a_{j}$ and $b_{j}$, there exists a non-negative integer number $k$ such that the operator $W$ can be written in the form

$$
W=U+\widetilde{U},
$$

where the operator $U$ is comprised of at most four terms whose coefficients each involve the parameter $\mu$ to a power not exceeding $k+1$, and all terms of the operator $\widetilde{U}$ contain a factor $\mu$ at a power at least equal to $k+2$. The operator $U$ has the following form:

$$
\begin{aligned}
(U f)(t)= & \chi_{\left[\alpha^{\prime}, \alpha^{\prime}+\alpha_{1}\right]}(t) \\
& \times\left[c_{1} f\left(t-w_{1}\right)+c_{2} f\left(t-w_{1}-\alpha_{1}\right)\right. \\
& \left.+c_{3} f\left(t-w_{2}+\alpha_{1}\right)+c_{4} f\left(t-w_{2}\right)\right],
\end{aligned}
$$

where one or more of the coefficients $c_{i}$ may be zero.

Note that the operator $U$ may contain 2, 3 or 4 terms depending on the locations and the lengths of the intervals. There are many cases and sub-cases of the position of the intervals, so the proof is postponed to Appendix A. Exact formulae for the operator $U$, which are important for the proof of the invertibility of $U$ and $W$, are derived in the process of the proof.

We prove that for sufficiently small $\mu$ the invertibility of the operator $U$ implies that of the operator $W$. This statement is based on the following lemma, which is proved in Appendix $B$.

Lemma 1. If the operator $U$ is invertible, then $\left\|U^{-1}\right\| \leq$ $|\mu|^{-(k+1)} C$ for $|\mu|$ small enough, where $C>0$ does not depend on $\mu$.

Theorem 3. If the operator $U$ is invertible, then for sufficiently small $\mu$, the operator $W$ is also invertible.
Proof. Theorem 2 states that the operator $W$ can be represented as a sum of two other operators $W=U+\widetilde{U}$. The operator $U$ is made up of no more than 4 terms of $W$ with powers less than or equal to $k+1$, and the operator $\widetilde{U}$ contains the rest of the terms of $W$.

We have noticed that the operator $W$ has a finite number of terms. Therefore, $\widetilde{U}$ also has a finite number of terms. Since $\widetilde{\widetilde{U}}$ contains only powers of $\mu$ higher than $k+1$, then for sufficiently small $\mu$ we have

$$
\|\widetilde{U}\| \leq|\mu|^{k+2} D
$$

where $D$ does not depend on $\mu$.

Then from Lemma 1 it follows that, for sufficiently small $\mu$,

$$
\left\|U^{-1}\right\|\|\widetilde{U}\|<1
$$

Note that

$$
W=U+\widetilde{U}=U\left(I+U^{-1} \widetilde{U}\right) .
$$

Thus, for sufficiently small $\mu$, the operator $W$ is invertible.

It is convenient to scale so that $\alpha_{1}=1$. After a change of variables the operator $U$ is reduced to the operator $V$ in $L^{2}(0,1)$ :

$$
\begin{aligned}
(V f)(t)= & \chi_{[0,1]}(t)\left[c_{1} f(t+a)+c_{2} f(t+a-1)\right. \\
& \left.+c_{3} f(t+b)+c_{4} f(t+b-1)\right] .
\end{aligned}
$$

Here

$$
0 \leq b \leq a \leq 1
$$

and $c_{i}$ are the corresponding coefficients $A(r, k, q)$ or 0 .

To prove that the operator $V$ is invertible, we introduce a new operator $K$ which has the same form as the operator $V$, but the coefficients $c_{i}$ are arbitrary real numbers. We consider two cases: the case when $a-b$ is a rational number and the case of irrational $a-b$. The case of $a-b \in \mathbb{Q}$ corresponds to the situation of $\alpha_{1} / \alpha_{2} \in \mathbb{Q}$ and the irrational case occurs if $\alpha_{1} / \alpha_{2} \in \mathbb{R} \backslash \mathbb{Q}$, where $\alpha_{1}$ and $\alpha_{2}$ are the lengths of the intervals $I_{1}$ and $I_{2}$ as in (7b). First, we find the invertibility condition for the operator $K$, and then we show that the coefficients $c_{i}$ of the operator $V$ satisfy this condition.

\section{Invertibility of the Operator $K$}

Consider the operator $K$ in $L^{2}[0,1]$ :

$$
\begin{aligned}
(K f)(t)= & {\left[c_{1} f(t+a)+c_{2} f(t+a-1)\right.} \\
& \left.+c_{3} f(t+b)+c_{4} f(t+b-1)\right]
\end{aligned}
$$

where $t \in[0,1] ; a, b \in[0,1] ; b \leq a ; c_{1} \neq 0$ or $c_{4} \neq 0$. Our goal is a sufficient condition for the invertibility of $K$. 
We do not consider the case of $c_{1}=c_{4}=0$ : the invertibility conditions for $K$ in this case are different from those in all other cases, and we do not need the case of $c_{1}=c_{4}=0$ to prove the invertibility of the operator $V$.

From (11) one can easily see that the invertibility of the operator $K$ is equivalent to the solvability for $f$ of the following system of equations:

$$
\begin{aligned}
c_{1} f(t+a)+c_{3} f(t+b) & =g(t), & & t \in[0,1-a), \\
c_{3} f(t+b)+c_{2} f(t+a-1) & =g(t), & & t \in(1-a, 1-b), \\
c_{2} f(t+a-1)+c_{4} f(t+b-1) & =g(t), & & t \in(1-b, 1],
\end{aligned}
$$

where $g(t)$ is in $L^{2}[0,1]$.

Let us find the conditions for the invertibility of the operator $K$ in special cases: $c_{1}=c_{3}=0, c_{2}=c_{3}=0$, $c_{2}=c_{4}=0$. The following lemma is a particular case of Theorems 4 and 5, which are proved in Subsections 3.1 and 3.2, respectively. We formulate it as a separate lemma because its proof is different from the proofs of these theorems.

Lemma 2. Suppose that $c_{1}=c_{3}=0$, or $c_{2}=c_{3}=0$, or $c_{2}=c_{4}=0$. If $a-b$ is a rational number, the operator $K$ is invertible in $L^{2}[0,1]$ if and only if

$$
(-1)^{n} c_{2}^{k_{1}+m} c_{1}^{n-m-k_{1}} \neq c_{3}^{n-k_{1}} c_{4}^{k_{1}}
$$

and

$$
(-1)^{n} c_{2}^{k_{2}+m} c_{1}^{n-m-k_{2}} \neq c_{3}^{n-k_{2}} c_{4}^{k_{2}},
$$

where $a-b=m / n, k_{1}$ is the integer part of $b n$, and $k_{2}$ is the smallest integer such that $k_{2} \geq b n$. If $a-b$ is an irrational number, the operator $K$ is invertible if and only if

$$
\left|c_{3}\right|^{1-b}\left|c_{4}\right|^{b} \neq\left|c_{2}\right|^{a}\left|c_{1}\right|^{1-a}
$$

Proof. Assume that $c_{1}=c_{3}=0$. If $a \neq 1$, the first equation of the system (12) becomes

$$
0=g(t), \quad t \in[0,1-a) .
$$

Consequently, the operator $K$ is not invertible.

If $a=1$, then the first equation of (12) is defined on an interval of length zero. We get a system of two equations; this system is solvable when $c_{2} \neq 0$ if $b \neq$ 1 , and when $c_{2}+c_{4} \neq 0$ if $b=1$. We can find the invertibility conditions for the cases of $c_{2}=c_{3}=0$ and $c_{2}=c_{4}=0$ using the same reasoning. We summarize all the cases in Table 1 . They can be generalized by the two conditions given in the statement of this lemma.

In what follows, we will need to divide the equations of the system (12) by $c_{1}$ and $c_{2}$ or $c_{3}$ and $c_{4}$. By proving Lemma 2, we exclude from consideration the cases when $c_{1}=c_{3}=0, c_{2}=c_{3}=0, c_{2}=c_{4}=0$. Thus we can assume that $\left(c_{1} \neq 0\right.$ and $\left.c_{2} \neq 0\right)$ or $\left(c_{3} \neq 0\right.$ and $\left.c_{4} \neq 0\right)$.
Table 1. Cases considered in the proof of Lemma 2

\begin{tabular}{|l|l|}
\hline \multicolumn{1}{|c|}{ Coefficients } & Invertibility condition \\
\hline \hline$c_{1}=c_{3}=0, \quad a \neq 1$ & not invertible \\
$c_{1}=c_{3}=0, \quad a=1, b \neq 1$ & $c_{2} \neq 0$ \\
$c_{1}=c_{3}=0, \quad a=b=1$ & $c_{2}+c_{4} \neq 0$ \\
\hline$c_{2}=c_{3}=0, \quad a \neq b$ & not invertible \\
$c_{2}=c_{3}=0, \quad a=b, a \neq 1, a \neq 0$ & $c_{1} \neq 0$ and $c_{4} \neq 0$ \\
$c_{2}=c_{3}=0, \quad a=b=0$ & $c_{1} \neq 0$ \\
$c_{2}=c_{3}=0, \quad a=b=1$ & $c_{4} \neq 0$ \\
\hline$c_{2}=c_{4}=0, \quad b \neq 0$ & not invertible \\
$c_{2}=c_{4}=0, \quad b=0, a \neq 0$ & $c_{3} \neq 0$ \\
$c_{2}=c_{4}=0, \quad a=b=0$ & $c_{1}+c_{3} \neq 0$ \\
\hline
\end{tabular}

Let $\Delta=a-b, \bar{\Delta}=1-\Delta$. If $c_{3} \neq 0$ and $c_{4} \neq 0$, then the system (12) is equivalent to

$$
\begin{aligned}
f(t)+\frac{c_{1}}{c_{3}} f(t+\Delta) & =\frac{1}{c_{3}} g(t-b), & t \in[b, \bar{\Delta}), \\
f(t)+\frac{c_{2}}{c_{3}} f(t+\Delta-1) & =\frac{1}{c_{3}} g(t-b), & t \in(\bar{\Delta}, 1), \\
f(t)+\frac{c_{2}}{c_{4}} f(t+\Delta) & =\frac{1}{c_{4}} g(t+1-b), & t \in(0, b],
\end{aligned}
$$

which, in turn, is equivalent to

$$
f(t)+\phi(t) f((t+\Delta) \bmod 1)=h(t), \quad t \in[0,1],
$$

where

$$
\phi(t)= \begin{cases}c_{2} / c_{4}, & t \in(0, b), \\ c_{1} / c_{3}, & t \in(b, \bar{\Delta}), \\ c_{2} / c_{3}, & t \in(\bar{\Delta}, 1) .\end{cases}
$$

If $c_{1} \neq 0$ and $c_{2} \neq 0$, the system (12) amounts to

$$
f(t)+\psi(t) f((t+\bar{\Delta}) \bmod 1)=k(t), \quad t \in[0,1],
$$

where

$$
\psi(t)= \begin{cases}c_{3} / c_{2}, & t \in(0, \Delta), \\ c_{4} / c_{2}, & t \in(\Delta, a), \\ c_{3} / c_{1}, & t \in(a, 1) .\end{cases}
$$

Equations of the type (13) were investigated in (Antonevich, 1996, Thm. 2.1, pp. 29-32) for the case of continuous $\phi(t)$. In the course of the proof of Theorems 4 and 5 we will obtain solvability conditions for (13) and (15) for piecewise continuous $\phi(t)$ and $\psi(t)$ as defined in (14) and (16).

\subsection{Case of $a-b \in \mathbb{Q}$}

Theorem 4. Let $a-b=m / n$ be an irreducible fraction. The operator $K$ is invertible in $L^{2}[0,1]$ if and only if

$$
(-1)^{n} c_{2}^{k_{1}+m} c_{1}^{n-m-k_{1}} \neq c_{3}^{n-k_{1}} c_{4}^{k_{1}}
$$


and

$$
(-1)^{n} c_{2}^{k_{2}+m} c_{1}^{n-m-k_{2}} \neq c_{3}^{n-k_{2}} c_{4}^{k_{2}},
$$

where $k_{1}$ is the integer part of bn, and $k_{2}$ is the smallest integer such that $k_{2} \geq$ bn.

The results equivalent to Theorem 4 were independently obtained by Spitkovsky (2006). The theory of convolution operators in spaces of matrix valued functions can be found in the book (Böttcher et al., 2002).

Proof. From Lemma 2 it follows that this theorem holds for the cases $c_{1}=c_{3}=0, c_{2}=c_{3}=0, c_{2}=c_{4}=0$. Thus we do not have to consider these cases in the proof of Theorem 4. We defined the operator $K$ so that $c_{1} \neq 0$ or $c_{4} \neq 0$. Since we excluded the cases of $c_{1}=c_{3}=0$, $c_{2}=c_{3}=0, c_{2}=c_{4}=0$, we may assume that $\left(c_{1} \neq 0\right.$ or $\left.c_{3} \neq 0\right)$ and $\left(c_{2} \neq 0\right.$ or $\left.c_{3} \neq 0\right)$ and $\left(c_{2} \neq 0\right.$ or $\left.c_{4} \neq 0\right)$. That means that $\left(c_{1} \neq 0\right.$ and $\left.c_{2} \neq 0\right)$ or $\left(c_{3} \neq 0\right.$ and $\left.c_{4} \neq 0\right)$.

As we have already noted, when both $c_{3}$ and $c_{4}$ are not equal to zero, the invertibility of the operator $K$ is equivalent to the solvability of (13). If $c_{3}=0$ or $c_{4}=0$ then $c_{1} \neq 0$ and $c_{2} \neq 0$, and in this case the invertibility of $K$ is equivalent to the solvability of (15). In the first case the problem will be reduced to the solvability of two algebraic systems with determinants:

$$
1+(-1)^{n+1} c_{2}^{k_{2}+m} c_{1}^{n-m-k_{2}} /\left(c_{3}^{n-k_{2}} c_{4}^{k_{2}}\right)
$$

and

$$
1+(-1)^{n+1} c_{2}^{k_{1}+m} c_{1}^{n-m-k_{1}} /\left(c_{3}^{n-k_{1}} c_{4}^{k_{1}}\right) .
$$

In the second case the problem reduces to the solvability of two systems with determinants:

$$
1+(-1)^{n+1} c_{3}^{n-k_{2}} c_{4}^{k_{2}} /\left(c_{2}^{k_{2}+m} c_{1}^{n-m-k_{2}}\right)
$$

and

$$
1+(-1)^{n+1} c_{3}^{n-k_{1}} c_{4}^{k_{1}} /\left(c_{2}^{k_{1}+m} c_{1}^{n-m-k_{1}}\right) .
$$

The proofs of the last two facts are analogous, so we will only show the derivation of the first of them.

Suppose that $c_{3} \neq 0$ and $c_{4} \neq 0$. Let us rewrite (13) as a family of equations defined on disjoint subintervals of the interval $[0,1]$, choosing subintervals so that in each of those equations the function $\phi(t)$ is constant.

First we divide the interval into $n$ pieces of the length $1 / n:\{((i-1) / n, i / n)\}_{i=1}^{n}$. The number of such subintervals that are entirely inside of the interval $[0, b]$ is equal to the integer part of $b n$. Let us denote this number by $k$. Let us also introduce $d$ as the length of the interval $[k / n, b]: d=b-k / n$.

Now we divide each subinterval of the length $1 / n$ into two smaller subintervals with the lengths of $d$ and
$1 / n-d$, and consider two sets of subintervals:

$$
\begin{aligned}
& J_{1}=\left\{\left(\frac{i-1}{n}, \frac{i-1}{n}+d\right)\right\}_{i=1}^{n}, \\
& J_{2}=\left\{\left(\frac{i-1}{n}+d, \frac{i}{n}\right)\right\}_{i=1}^{n} .
\end{aligned}
$$

The set $J_{1}$ contains all intervals of the length $d$, and $J_{2}$ has all intervals of the length $1 / n-d$.

Note that $\phi(t)$ is constant on each of these subintervals $(\phi(t)$ is piecewise constant and it changes its values at the points $b=k / n+d$ and $\bar{\Delta}=(n-m) / n)$.

Now we can rewrite (13) as the following family of equations:

$$
\begin{aligned}
& f(t)+c_{2} c_{4}^{-1} f(t+\Delta)=h(t), \quad t \in(0, d), \\
& f(t)+c_{2} c_{4}^{-1} f(t+\Delta)=h(t), \quad t \in\left(d, \frac{1}{n}\right), \\
& f(t)+c_{2} c_{4}^{-1} f(t+\Delta)=h(t), \quad t \in\left(\frac{k-1}{n}+d, \frac{k}{n}\right), \\
& f(t)+c_{2} c_{4}^{-1} f(t+\Delta)=h(t), \\
& t \in\left(\frac{k}{n}, \frac{k}{n}+d\right)=\left(\frac{k}{n}, b\right), \\
& f(t)+c_{1} c_{3}^{-1} f(t+\Delta)=h(t), \\
& t \in\left(\frac{k}{n}+d, \frac{k+1}{n}\right)=\left(b, \frac{k+1}{n}\right), \\
& f(t)+c_{1} c_{3}^{-1} f(t+\Delta)=h(t), t \in\left(\frac{k+1}{n}, \frac{k+1}{n}+d\right), \\
& f(t)+c_{1} c_{3}^{-1} f(t+\Delta)=h(t) \\
& t \in\left(\frac{n-m-1}{n}, \frac{n-m-1}{n}+d\right), \\
& f(t)+c_{1} c_{3}^{-1} f(t+\Delta)=h(t), t \in\left(\frac{n-m-1}{n}+d, \bar{\Delta}\right), \\
& f(t)+c_{2} c_{3}^{-1} f(t+\Delta-1)=h(t), t \in\left(\bar{\Delta}, \frac{n-m}{n}+d\right), \\
& f(t)+c_{2} c_{3}^{-1} f(t+\Delta-1)=h(t), \\
& t \in\left(\frac{n-m}{n}+d, \frac{n-m+1}{n}\right) \\
& f(t)+c_{2} c_{3}^{-1} f(t+\Delta-1)=h(t) \\
& t \in\left(\frac{n-1}{n}, \frac{n-1}{n}+d\right), \\
& f(t)+c_{2} c_{3}^{-1} f(t+\Delta-1)=h(t), t \in\left(\frac{n-1}{n}+d, 1\right) .
\end{aligned}
$$

Note that this family has three groups of equations: the first group contains $2 k+1$ equations defined on subintervals of $(0, b)$, and the coefficient of $f(t+\Delta)$ is $c_{2} / c_{4}$; the 
second group contains $2 n-2 m-2 k-1$ equations with the coefficient of $f(t+\Delta)$ equal to $c_{1} / c_{3}$; in the third group there are $2 m$ equations, and the coefficient is $c_{2} / c_{3}$.

Let us introduce $f_{i}, g_{i} \in L_{2}(0,1 / n)$ for $1 \leq i \leq n$ :

$$
\begin{aligned}
f_{i}(t) & =f\left(t+\frac{i-1}{n}\right), \\
h_{i}(t) & =h\left(t+\frac{i-1}{n}\right) .
\end{aligned}
$$

Replacing $f(t)$ and $h(t)$ by $f_{i}(t)$ and $h_{i}(t)$, respectively, in each of the equations of the family (17), the latter can be transformed into two systems:

$$
\left\{\begin{aligned}
f_{1}(t)+c_{2} c_{4}^{-1} f_{m+1}(t) & =h_{1}(t), \\
& \vdots \\
f_{k+1}(t)+c_{2} c_{4}^{-1} f_{k+m+1}(t) & =h_{k+1}(t), \\
f_{k+2}(t)+c_{1} c_{3}^{-1} f_{k+m+2}(t) & =h_{k+2}(t), \\
& \vdots \\
f_{n-m}(t)+c_{1} c_{3}^{-1} f_{n}(t) & =h_{n-m}(t), \\
f_{n-m+1}(t)+c_{2} c_{3}^{-1} f_{1}(t) & =h_{n-m+1}(t), \\
& \vdots \\
f_{n}(t)+c_{2} c_{3}^{-1} f_{m}(t) & =h_{n}(t),
\end{aligned}\right.
$$

on $t \in(0, d)$;

$$
\left\{\begin{aligned}
f_{1}(t)+c_{2} c_{4}^{-1} f_{m+1}(t) & =h_{1}(t), \\
& \vdots \\
f_{k}(t)+c_{2} c_{4}^{-1} f_{k+m}(t) & =h_{k}(t), \\
f_{k+1}(t)+c_{1} c_{3}^{-1} f_{k+m+1}(t) & =h_{k+1}(t), \\
& \vdots \\
f_{n-m}(t)+c_{1} c_{3}^{-1} f_{n}(t) & =h_{n-m}(t), \\
f_{n-m+1}(t)+c_{2} c_{3}^{-1} f_{1}(t) & =h_{n-m+1}(t), \\
& \vdots \\
f_{n}(t)+c_{2} c_{3}^{-1} f_{m}(t) & =h_{n}(t)
\end{aligned}\right.
$$

on $t \in(d, 1 / n)$.

Let $x_{i}(t)=f_{(1+(i-1) m) \bmod n}(t)$. Since $m$ and $n$ are co-prime, this substitution maps the set $\left\{f_{i}(t)\right\}_{i=1}^{n}$ into the set $\left\{x_{i}(t)\right\}_{i=1}^{n}$.

The systems (18) and (19) take the forms

$$
x_{i}(t)+\psi_{i} x_{(i+1) \bmod n}(t)=h_{i}(t)
$$

on $t \in(0, d), 1 \leq i \leq n$;

$$
x_{i}(t)+\xi_{i} x_{(i+1) \bmod n}(t)=h_{i}(t)
$$

on $t \in(d, 1 / n), 1 \leq i \leq n$, where

$$
\psi_{i}, \xi_{i} \in\left\{c_{2} / c_{4}, c_{1} / c_{3}, c_{2} / c_{3}\right\}, \quad 1 \leq i \leq n .
$$

In the system (20), $\left\{\psi_{i}\right\}_{i=1}^{n}$ has $k+1$ occurrences of $c_{2} / c_{4}, n-m-k-1$ occurrences of $c_{1} / c_{3}$, and $m$ occurrences of $c_{2} / c_{3}$. The system (21) has $k$ occurrences of $c_{2} / c_{4}, n-m-k$ occurrences of $c_{1} / c_{3}$, and $m$ occurrences of $c_{2} / c_{3}$. Therefore, the determinant of the first system is equal to

$$
\begin{aligned}
1+(-1)^{n+1} & \prod_{i=1}^{n} \psi_{i} \\
& =1+(-1)^{n+1}\left(\frac{c_{2}}{c_{4}}\right)^{k+1}\left(\frac{c_{1}}{c_{3}}\right)^{n-m-k-1}\left(\frac{c_{2}}{c_{3}}\right)^{m} \\
& =1+(-1)^{n+1} \frac{c_{2}^{k+m+1} c_{1}^{n-m-k-1}}{c_{3}^{n-k-1} c_{4}^{k+1}}
\end{aligned}
$$

The determinant of the second system is

$$
1+(-1)^{n+1} \prod_{i=1}^{n} \xi_{i}=1+(-1)^{n+1} \frac{c_{2}^{k+m} c_{1}^{n-m-k}}{c_{3}^{n-k} c_{4}^{k}} .
$$

If $b n$ is an integer, say, $b n=k$, then $d=0$, and the first system lives on an empty interval. In this case, the invertibility of the operator $K$ is equivalent to the solvability of the system (21) with a determinant:

$$
1+(-1)^{n+1} c_{2}^{k+m} c_{1}^{n-m-k} /\left(c_{3}^{n-k} c_{4}^{k}\right)(k=b n) .
$$

So $K$ is invertible if and only if

$$
1+(-1)^{n+1} c_{2}^{k+m} c_{1}^{n-m-k} /\left(c_{3}^{n-k} c_{4}^{k}\right) \neq 0 .
$$

Let $b n \neq k$. In this case, both intervals $(0, d)$ and $(d, 1 / n)$ are non-empty. Therefore, the invertibility of $K$ is equivalent to non-zero determinants:

$$
1+(-1)^{n+1} c_{2}^{k+m+1} c_{1}^{n-m-k-1} /\left(c_{3}^{n-k-1} c_{4}^{k+1}\right)
$$

and

$$
1+(-1)^{n+1} c_{2}^{k+m} c_{1}^{n-m-k} /\left(c_{3}^{n-k} c_{4}^{k}\right) .
$$

Here $k$ is the integer part of $b n$, and $k+1$ is the least integer greater than or equal to $b n$.

In the formulation of Theorem 4 we defined $k_{1}$ as the integer part of $b n$, and $k_{2}$ as the smallest integer such that $k_{2} \geq b n$. Now we can see that the determinants (23) and (22) are equal to

$$
1+(-1)^{n+1} c_{2}^{k_{1}+m} c_{1}^{n-m-k_{1}} /\left(c_{3}^{n-k_{1}} c_{4}^{k_{1}}\right)
$$

and

$$
1+(-1)^{n+1} c_{2}^{k_{2}+m} c_{1}^{n-m-k_{2}} /\left(c_{3}^{n-k_{2}} c_{4}^{k_{2}}\right),
$$


respectively, and they are not equal to zero when

$$
(-1)^{n} c_{2}^{k_{1}+m} c_{1}^{n-m-k_{1}} \neq c_{3}^{n-k_{1}} c_{4}^{k_{1}}
$$

and

$$
(-1)^{n} c_{2}^{k_{2}+m} c_{1}^{n-m-k_{2}} \neq c_{3}^{n-k_{2}} c_{4}^{k_{2}} .
$$

This proves the theorem for the case of $c_{3} \neq 0$ and $c_{4} \neq 0$.

In the beginning of the proof we argued that, in the case of $c_{3}=0$ or $c_{4}=0$, the invertibility of $K$ amounts to nonzero values of

$$
1+(-1)^{n+1} c_{3}^{n-k_{2}} c_{4}^{k_{2}} /\left(c_{2}^{k_{2}+m} c_{1}^{n-m-k_{2}}\right)
$$

and

$$
1+(-1)^{n+1} c_{3}^{n-k_{1}} c_{4}^{k_{1}} /\left(c_{2}^{k_{1}+m} c_{1}^{n-m-k_{1}}\right) .
$$

They do not equal zero if and only if

$$
(-1)^{n} c_{2}^{k_{1}+m} c_{1}^{n-m-k_{1}} \neq c_{3}^{n-k_{1}} c_{4}^{k_{1}}
$$

and

$$
(-1)^{n} c_{2}^{k_{2}+m} c_{1}^{n-m-k_{2}} \neq c_{3}^{n-k_{2}} c_{4}^{k_{2}} .
$$

The theorem is thus proved.

\subsection{Case of $a-b \in \mathbb{R} \backslash \mathbb{Q}$}

Theorem 5. When $a-b$ is irrational, the operator $K$ is invertible if

$$
\left|c_{3}\right|^{1-b}\left|c_{4}\right|^{b} \neq\left|c_{2}\right|^{a}\left|c_{1}\right|^{1-a} .
$$

Notice that, following the scheme of the proof in (Antonevich, 1996, Thm. 2.1, pp. 29-32), it is possible to show that the above condition is necessary and sufficient for the invertibility of the operator $K$. We omit the proof of the "necessary" part since we do not use it in the application to sampling and interpolation problems.

Proof. From Lemma 2 it follows that this theorem holds for the cases $c_{1}=c_{3}=0, c_{2}=c_{3}=0, c_{2}=c_{4}=0$. Thus we do not have to consider these cases to prove this theorem. This means (see the beginning of the proof of Theorem 4$)$ we can assume that $\left(c_{1} \neq 0\right.$ and $\left.c_{2} \neq 0\right)$ or $\left(c_{3} \neq 0\right.$ and $\left.c_{4} \neq 0\right)$.

We know that when $c_{3} \neq 0$ and $c_{4} \neq 0$, the operator $K$ is invertible if (13) has a unique solution. If $c_{3}=0$ or $c_{4}=0$, we can assume that $c_{1} \neq 0$ and $c_{2} \neq 0$, and in this case $K$ is invertible when (15) has a unique solution. In this proof we first consider the case of $c_{3} \neq 0, c_{4} \neq 0$ and $\left|c_{2}\right|^{a}\left|c_{1}\right|^{1-a}\left|c_{3}\right|^{b-1}\left|c_{4}\right|^{-b}<1$. Next we turn to the proof for $\left|c_{2}\right|^{a}\left|c_{1}\right|^{1-a}\left|c_{3}\right|^{b-1}\left|c_{4}\right|^{-b}>1$ and for $c_{3}=0$ or $c_{4}=0$; it runs on almost the same lines and leads to the same result.
Suppose that $c_{3} \neq 0, \quad c_{4} \neq 0$ and $\left|c_{2}\right|^{a}\left|c_{1}\right|^{1-a}\left|c_{3}\right|^{b-1}\left|c_{4}\right|^{-b}<1$. In this case, the invertibility of the operator $K$ is equivalent to the solvability of Eqn. (13):

$$
f(t)+\phi(t) f((t+\Delta) \bmod 1)=h(t), \quad t \in[0,1],
$$

where

$$
\phi(t)= \begin{cases}c_{2} / c_{4}, & t \in(0, b), \\ c_{1} / c_{3}, & t \in(b, \bar{\Delta}), \\ c_{2} / c_{3}, & t \in(\bar{\Delta}, 1) .\end{cases}
$$

To solve (13), we can apply successive approximations:

$$
\begin{aligned}
f_{0}(t) & =h(t), \\
f_{n+1}(t) & =-\phi(t) f_{n}((t+\Delta) \bmod 1)+h(t), \\
& n=0,1, \ldots .
\end{aligned}
$$

Then

$$
\begin{aligned}
& f_{n+1}(t)-f_{n}(t) \\
& =\left(\prod_{j=0}^{n-1}[-\phi((t+j \Delta) \bmod 1)]\right) \\
& \quad \times\left[f_{1}((t+n \Delta) \bmod 1)-f_{0}((t+n \Delta) \bmod 1)\right] .
\end{aligned}
$$

If $c_{1}=0$ or $c_{2}=0$, then there is $l$ such that

$$
f_{n+1}(t)-f_{n}(t)=0 \quad \text { for any } n \geq l .
$$

Thus, $f_{l}(t)$ is a solution to (13). Therefore, the operator $K$ is invertible when $c_{1}$ or $c_{2}$ is equal to zero, and $c_{3}$ and $c_{4}$ are both non-zero.

Now, let us assume that $c_{1} \neq 0$ and $c_{2} \neq 0$. Since $\ln |\phi(t)|$ is Riemann integrable, for any irrational $\Delta$ we have

$$
\begin{array}{r}
\frac{1}{N} \sum_{k=0}^{N-1} \ln |\phi((t+k \Delta) \bmod 1)| \underset{N \rightarrow \infty}{\longrightarrow} \int_{0}^{1} \ln |\phi(t)| \mathrm{d} t \\
=b \ln \left|\frac{c_{2}}{c_{4}}\right|+(1-a) \ln \left|\frac{c_{1}}{c_{3}}\right|+(a-b) \ln \left|\frac{c_{2}}{c_{3}}\right|,
\end{array}
$$

uniformly in $t \in[0,1]$ (see, e.g., (Peterson, 1983), p. 156). Then

$$
\begin{aligned}
& \lim _{N \rightarrow \infty} \max _{t}\left(\prod_{j=0}^{N-1}|\phi((t+j \Delta) \bmod 1)|\right)^{1 / N} \\
& =\exp \lim _{N \rightarrow \infty} \max _{t} \frac{1}{N} \sum_{k=0}^{N-1} \ln |\phi((t+k \Delta) \bmod 1)| \\
& =\exp \left(b \ln \left|\frac{c_{2}}{c_{4}}\right|+(1-a) \ln \left|\frac{c_{1}}{c_{3}}\right|+(a-b) \ln \left|\frac{c_{2}}{c_{3}}\right|\right) \\
& =\left|\frac{c_{2}}{c_{4}}\right|^{b}\left|\frac{c_{1}}{c_{3}}\right|^{1-a}\left|\frac{c_{2}}{c_{3}}\right|^{a-b} .
\end{aligned}
$$


Note that

$$
\left|\frac{c_{2}}{c_{4}}\right|^{b}\left|\frac{c_{1}}{c_{3}}\right|^{1-a}\left|\frac{c_{2}}{c_{3}}\right|^{a-b}=\frac{\left|c_{2}\right|^{a}\left|c_{1}\right|^{1-a}}{\left|c_{3}\right|^{1-b}\left|c_{4}\right|^{b}}<1 .
$$

Then, for any $\varepsilon$ such that

$$
\left|\frac{c_{2}}{c_{4}}\right|^{b}\left|\frac{c_{1}}{c_{3}}\right|^{1-a}\left|\frac{c_{2}}{c_{3}}\right|^{a-b}<\varepsilon<1,
$$

there exists $M$ such that

$$
\max _{t}\left(\prod_{j=0}^{N-1}|\phi((t+j \Delta) \bmod 1)|\right)^{1 / N}<\varepsilon
$$

for any $N \geq M$.

Then, from (24) and (25), we obtain that, for sufficiently large $n$,

$$
\begin{aligned}
\mid f_{n+p}- & f_{n} \mid \\
& \leq \sum_{k=1}^{p} \prod_{j=0}^{n+k-2}|\phi((t+j \Delta) \bmod 1)| \\
& \times \mid f_{1}((t+(n+k-1) \Delta) \bmod 1) \\
& -f_{0}((t+(n+k-1) \Delta) \bmod 1) \mid
\end{aligned}
$$

and

$$
\begin{aligned}
\left\|f_{n+p}-f_{n}\right\|_{L^{2}}^{2} & \leq 2 \sum_{k=1}^{\infty}\left(\varepsilon^{n+k-1}\right)^{2}\left\|f_{1}-f_{0}\right\|_{L^{2}}^{2} \\
& =\frac{2 \varepsilon^{2 n}}{1-\varepsilon^{2}}\left\|f_{1}-f_{0}\right\|_{L^{2}}^{2} .
\end{aligned}
$$

Thus,

$$
\left\|f_{n+p}-f_{n}\right\|_{L^{2}} \leq \frac{\sqrt{2} \varepsilon^{n}}{\sqrt{1-\varepsilon^{2}}}\left\|f_{1}-f_{0}\right\|_{L^{2}} .
$$

The norm $\left\|f_{n+p}-f_{n}\right\|_{L^{2}}$ can be made arbitrarily small for all $p$ by taking sufficiently large $n$. Therefore, the sequence $\left\{f_{i}\right\}_{i=1}^{n}$ converges to a function $f$, and $f(t)$ is the solution to (13).

Suppose now that $c_{1} \neq 0, c_{2} \neq 0$ and $\left|c_{4}\right|^{b}\left|c_{3}\right|^{1-b}\left|c_{1}\right|^{a-1}\left|c_{2}\right|^{-a}<1$. Whenever $c_{1} \neq 0$ and $c_{2} \neq 0$, the invertibility of the operator $K$ is equivalent to the solvability of (15):

$$
f(t)+\psi(t) f((t+\bar{\Delta}) \bmod 1)=k(t), \quad t \in[0,1],
$$

where

$$
\psi(t)= \begin{cases}c_{3} / c_{2}, & t \in(0, \Delta), \\ c_{4} / c_{2}, & t \in(\Delta, a), \\ c_{3} / c_{1}, & t \in(a, 1) .\end{cases}
$$

This time we use the same kind of successive approximations to prove the solvability of the second equation (15). We use the fact that

$$
\begin{aligned}
\lim _{n \rightarrow \infty} \max _{t}\left(\prod_{j=0}^{N-1}|\psi((t+j \bar{\Delta}) \bmod 1)|\right)^{1 / N} \\
=\left|\frac{c_{4}}{c_{2}}\right|^{b}\left|\frac{c_{3}}{c_{1}}\right|^{1-a}\left|\frac{c_{3}}{c_{2}}\right|^{a-b}<1
\end{aligned}
$$

to prove that the new sequence $\left\{f_{i}\right\}$ converges to the solution of (15).

Therefore, the operator $K$ is invertible when $\left|c_{3}\right|^{1-b}\left|c_{4}\right|^{b} \neq\left|c_{2}\right|^{a}\left|c_{1}\right|^{1-a}$.

\section{Invertibility of the Operator $V$}

In this section we use Theorems 4 and 5 to show that the operator $V$ (see (10)) is invertible.

From (35)-(44) (see the Appendix), we know that there are three kinds of the operator $V$ : (a) with $c_{1} \neq 0$, $c_{2} \neq 0$, and $c_{3}=c_{4}=0$ (or $c_{3} \neq 0, c_{4} \neq 0$, and $c_{1}=c_{2}=0$ ); (b) with only one of the coefficients $c_{i}$ equal to zero; (c) with $c_{i} \neq 0$ for $1 \leq i \leq 4$.

In Case 1 the conditions of Theorems 4 and 5 hold, and hence the operator $V$ is invertible.

Let us prove that if only one of the coefficients $c_{i}$ is equal to zero, the operator $V$ is invertible.

Theorem 6. When exactly one of the coefficients $c_{i}$ is equal to zero, the operator $V$ is invertible in $L^{2}[0,1]$ for sufficiently small $\mu$.

Proof. The conditions of Theorems 4 and 5 hold if the zero coefficient is raised to a non-zero power. For example, if $a-b$ is an irrational number, $c_{3}=0$, and $b \neq 1$, then the condition of Theorem 5 becomes $|0|^{1-b}\left|c_{4}\right|^{b} \neq$ $\left|c_{2}\right|^{a}\left|c_{1}\right|^{1-a}$, or $\left|c_{2}\right|^{a}\left|c_{1}\right|^{1-a} \neq 0$, which is obviously true.

We will have to separately handle the cases when the zero coefficient is raised to the zeroth power. In case $a-$ $b \in \mathbb{R} \backslash \mathbb{Q}, c_{1}=0$ and $a=1$, the condition of Theorem 5 becomes

$$
\left|c_{3}\right|^{1-b}\left|c_{4}\right|^{b} \neq\left|c_{2}\right| .
$$

In case $a-b \in \mathbb{R} \backslash \mathbb{Q}, c_{4}=0$ and $b=0$, the condition of Theorem 5 becomes

$$
\left|c_{3}\right| \neq\left|c_{2}\right|^{a}\left|c_{1}\right|^{1-a}
$$

From the formulae for the coefficients $c_{i}$ (36), (37), (40), (41) and (43) derived in the proof of Theorem 2 in Appendix A, it follows that the left and right-hand sides of the inequalities (26) and (27) involve different powers of $\mu$. Thus, for sufficiently small $\mu$, the inequalities (26) and (27) hold, and the operator $V$ is invertible. 
In case $a-b \in \mathbb{Q}$ and $c_{1}=0$, the condition of Theorem 4 is true unless $n-m-k_{1}=0$ or $n-m-k_{2}=0$. When $n-m-k_{i}=0$, the condition of Theorem 4 is

$$
(-1)^{n} c_{2}^{n} \neq c_{3}^{m} c_{4}^{n-m}
$$

In case $a-b \in \mathbb{Q}, c_{2}=0$ and $k_{i}+m=0$, the condition of Theorem 4 is

$$
(-1) c_{1} \neq c_{3}
$$

In case $a-b \in \mathbb{Q}, c_{3}=0$ and $n=k_{i}$, the condition of Theorem 4 is

$$
(-1) c_{2} \neq c_{4}
$$

In case $a-b \in \mathbb{Q}, c_{4}=0$ and $k_{i}=0$, the condition of Theorem 4 is

$$
(-1)^{n} c_{2}^{m} c_{1}^{n-m} \neq c_{3}^{n}
$$

One can check that if $m \neq 0$, the conditions (28) and (31) hold for sufficiently small $\mu$, since the left and right-hand sides of the inequalities involve different powers of $\mu$. Therefore, the operator $V$ is invertible in these cases.

If $m=0$, then $n=1$, since $m / n$ is an irreducible fraction. The inequalities (28) and (31) take the form of the inequalities (30) and (29), respectively. To show that the inequalities (29) and (30) hold, we use the fact that

$$
|A(r, k, 0)|>|A(r-k+1, k, k)| \quad \text { for } \quad k>1 .
$$

From the formulae for the coefficients $c_{i}$ (36), (37), (40), (41), (43) and the relation (32), it follows that one of the coefficients $c_{1}, c_{3}$ (or $c_{2}, c_{4}$ ) is larger than the other as far as the absolute value is concerned, or both coefficients are positive or negative. Thus, the inequalities (29) and (30) hold.

Let us consider the case of $c_{i} \neq 0$ for $1 \leq i \leq 4$. We have found the relation (42): In this case the operator $U$ is given by the following formula:

$$
\begin{aligned}
& (U f)(t) \\
& =\chi_{\left[\alpha^{\prime}, \alpha^{\prime}+\alpha_{1}\right]}(t)\left[A\left(r_{1}, k_{1}, 0\right) f\left(t-w\left(r_{1}, k_{1}, 0\right)\right)\right. \\
& +A\left(r_{1}, k_{1}+1,0\right) f\left(t-w\left(r_{1}, k_{1}+1,0\right)\right) \\
& +A\left(r_{1}-k_{1}+1, k_{1}, k_{1}\right) f\left(t-w\left(r_{1}-k_{1}+1, k_{1}, k_{1}\right)\right) \\
& +A\left(r_{1}-k_{1}, k_{1}+1, k_{1}+1\right) \\
& \left.\quad \times f\left(t-w\left(r_{1}-k_{1}, k_{1}+1, k_{1}+1\right)\right)\right]
\end{aligned}
$$

(see the proof of Theorem 2 in Appendix A).
From this relation we can see that coefficients $c_{i}$ can have two forms:

$$
\begin{aligned}
c_{1} & =A(r, k, 0)=(-1)^{r+k} \mu^{k} \frac{(r+k) !}{r ! k !}, \\
c_{2} & =A(r, k+1,0) \\
& =(-1)^{r+k+1} \mu^{k+1} \frac{(r+k+1) !}{r !(k+1) !}, \\
c_{3} & =A(r-k, k+1, k+1) \\
& =(-1)^{r+1} \mu^{k+1} \frac{(r+1) !}{(r-k) !(k+1) !} \\
c_{4} & =A(r-k+1, k, k) \\
& =(-1)^{r+1} \mu^{k} \frac{(r+1) !}{(r-k+1) ! k !},
\end{aligned}
$$

and

$$
\begin{aligned}
c_{1} & =A(r-k, k+1, k+1) \\
& =(-1)^{r+1} \mu^{k+1} \frac{(r+1) !}{(r-k) !(k+1) !}, \\
c_{2} & =A(r-k+1, k, k) \\
& =(-1)^{r+1} \mu^{k} \frac{(r+1) !}{(r-k+1) ! k !}, \\
c_{3} & =A(r, k, 0) \\
& =(-1)^{r+k} \mu^{k} \frac{(r+k) !}{r ! k !}, \\
c_{4} & =A(r, k+1,0) \\
& =(-1)^{r+k+1} \mu^{k+1} \frac{(r+k+1) !}{r !(k+1) !} .
\end{aligned}
$$

with some $r \geq 0$ and $k \geq 1$ (we do not have to consider cases with $k=0$, because when $k=0$, at least one of the coefficients $c_{i}$ is zero).

Theorem 7. When $a-b$ is rational and $c_{i} \neq 0,1 \leq i \leq 4$, the operator $V$ is invertible in $L^{2}[0,1]$ for a sufficiently small $\mu$.

Proof. By Theorem 4, the operator $V$ is invertible if and only if

$$
(-1)^{n} c_{2}^{k_{1}+m} c_{1}^{n-m-k_{1}} \neq c_{3}^{n-k_{1}} c_{4}^{k_{1}}
$$

and

$$
(-1)^{n} c_{2}^{k_{2}+m} c_{1}^{n-m-k_{2}} \neq c_{3}^{n-k_{2}} c_{4}^{k_{2}},
$$

where $a-b=m / n, k_{1}$ is the integer part of $b n$, and $k_{2}$ is the smallest integer such that $k_{2} \geq b n$.

From (33) and (34) we see that when $k_{1} \neq$ $(n-m) / 2$, the terms $c_{2}^{k_{1}+m} c_{1}^{n-m-k_{1}}$ and $c_{3}^{n-k_{1}} c_{4}^{k_{1}}$ have different powers of $\mu$. Therefore, in this case $\mu$ can be made small enough to make

$$
(-1)^{n} c_{2}^{k_{1}+m} c_{1}^{n-m-k_{1}} \neq c_{3}^{n-k_{1}} c_{4}^{k_{1}} .
$$


Similarly, when $k_{2} \neq(n-m) / 2$, for $\mu$ small enough,

$$
(-1)^{n} c_{2}^{k_{2}+m} c_{1}^{n-m-k_{2}} \neq c_{3}^{n-k_{2}} c_{4}^{k_{2}} .
$$

If $k_{1}$ or $k_{2}$ or both are equal to $(n-m) / 2$, then the corresponding inequality will have the form

$$
(-1)^{n} c_{2}^{\frac{n+m}{2}} c_{1}^{\frac{n-m}{2}} \neq c_{3}^{\frac{n+m}{2}} c_{4}^{\frac{n-m}{2}} .
$$

Now we cannot achieve the condition of Theorem 4 by making $\mu$ small, because the powers of $\mu$ are the same on both sides of the inequality. Consequently, we have to consider the specific forms of the coefficients $c_{i}$ (see (33) and (34)).

Since $k \geq 1$,

$$
|A(r, k, 0)| \geq|A(r-k+1, k, k)|
$$

and

$$
|A(r, k+1,0)|>|A(r-k, k+1, k+1)| .
$$

Then

$$
\left|c_{2}\right|^{\frac{n+m}{2}}\left|c_{1}\right|^{\frac{n-m}{2}} \neq\left|c_{3}\right|^{\frac{n+m}{2}}\left|c_{4}\right|^{\frac{n-m}{2}},
$$

and therefore

$$
(-1)^{n} c_{2}^{\frac{n+m}{2}} c_{1}^{\frac{n-m}{2}} \neq c_{3}^{\frac{n+m}{2}} c_{4}^{\frac{n-m}{2}}
$$

Theorem 8. When $a-b$ is irrational and $c_{i} \neq 0$ for $1 \leq$ $i \leq 4$, the operator $V$ is invertible in $L^{2}[0,1]$ for sufficiently small $\mu$.

Proof. From Theorem 5 we know that the operator $V$ is invertible if

$$
\left|c_{3}\right|^{1-b}\left|c_{4}\right|^{b} \neq\left|c_{2}\right|^{a}\left|c_{1}\right|^{1-a} .
$$

Using (33) and (34), we see that if $1-b \neq a$, then the terms $\left|c_{3}\right|^{1-b}\left|c_{4}\right|^{b}$ and $\left|c_{2}\right|^{a}\left|c_{1}\right|^{1-a}$ involve different powers of $\mu$. We can choose $\mu$ such that $\left|c_{3}\right|^{1-b}\left|c_{4}\right|^{b} \neq$ $\left|c_{2}\right|^{a}\left|c_{1}\right|^{1-a}$.

If $1-b=a$, then the expression above will have the form

$$
\left|c_{1}\right|^{1-a}\left|c_{2}\right|^{a} \neq\left|c_{3}\right|^{a}\left|c_{4}\right|^{1-a} .
$$

We will again have to look at the concrete forms of $c_{i}$. As we know, for $k \geq 1$,

$$
|A(r-k, k+1, k+1)|<|A(r, k+1,0)|
$$

and

$$
|A(r-k+1, k, k)| \leq|A(r, k, 0)| .
$$

Since $a-b=\Delta \in \mathbb{J}$ and $1-b=a$, we get $a \neq 0$ and $a \neq 1$. Thus, either

$$
\left|c_{1}\right|^{1-a}\left|c_{2}\right|^{a}<\left|c_{3}\right|^{a}\left|c_{4}\right|^{1-a}
$$

or

$$
\left|c_{1}\right|^{1-a}\left|c_{2}\right|^{a}>\left|c_{3}\right|^{a}\left|c_{4}\right|^{1-a} .
$$

Therefore,

$$
\left|c_{3}\right|^{1-b}\left|c_{4}\right|^{b} \neq\left|c_{2}\right|^{a}\left|c_{1}\right|^{1-a} .
$$

This completes the proof of the invertibility of the operator $V$ for irrational $\Delta$.

\section{Acknowledgments}

The authors are grateful to I. Spitkovsky for numerous fruitful discussions.

The research of Sergei Avdonin was partially supported by the National Science Foundation, Grant No. OPP-0414128, and by the Australian Research Council, Grant No. A00000723.

Anna Bulanova was partially supported by the University of Alaska Fairbanks Graduate Fellowship.

The research of William Moran was partially supported by the Australian Research Council, Grant No. A00000723.

\section{References}

Antonevich A. (1996): Linear Functional Equations. Operator Approach. — Basel, Birkhäuser.

Avdonin S. (1979): On Riesz bases from exponentials in $L^{2}$. Vestnik Leningrad Univ. Math., Vol. 7, pp. 203-211.

Avdonin S. and Ivanov S. (1995): Families of Exponentials. The Method of Moments in Controllability Problems for Distributed Parameter Systems. — New York: Cambridge University Press.

Avdonin S. and Moran W. (1999): Sampling and interpolation of functions with multi-band spectra and controllability problems, In: Optimal Control of Partial Differential Equations, (K.H. Hoffmann, G. Leugering and T. F., Eds.), Basel: Birkhäuser, Vol. 133, pp. 43-51.

Beaty M. and Dodson M. (1989): Derivative sampling for multiband signals. - Numer. Funct. Anal. Optim., Vol. 10, No. 9-10, pp. 875-898.

Beaty M. and Dodson M. (1993): The distribution of sampling rates for signals with equally wide, equally spaced spectral bands. - SIAM J. Appl. Math., Vol. 53, No. 3, pp. 893 906.

Bezuglaya L. and Katsnelson V. (1993): The sampling theorem for functions with limited multi-band spectrum, I. - Z . Anal. Anwendungen, Vol. 12, No. 3, pp. 511-534.

Böttcher A., Karlovich Y. and Spitkovsky I. (2002): Convolution Operators and Factorization of Almost Periodic Matrix Functions. — Basel: Birkhäuser.

Dodson M. and Silva A. (1989): An algorithm for optimal regular sampling. - Signal Process., Vol. 17, No. 2, pp. 169 174. 
Higgins J. (1996): Sampling Theory in Fourier and Signal Analysis: Foundations. — Oxford: Clarendon Press.

Hruščev S., Nikol'skii N. and Pavlov B. (1981): Unconditional bases of exponentials and reproducing kernels, In: Complex Analysis and Spectral Theory (V.P Havin and N.K. Nikol'ski, Eds.), Lecture Notes Math., Vol. 864, pp. 214335.

Katsnelson V. (1996): Sampling and interpolation for functions with multi-band spectrum: The mean-periodic continuation method, In: Wiener-Symposium (Grossbothen, 1994) Synerg. Syntropie Nichtlineare Syst., Vol. 4, Leipzig: Verlag Wiss. Leipzig, pp. 91-132,.

Kohlenberg A. (1953): Exact interpolation of band-limited functions. - J. Appl. Phys., Vol. 24, No. 12, pp. 1432-1436.

Lyubarskii Y. and Seip K. (1997): Sampling and interpolating sequences for multiband-limited functions and exponential bases on disconnected sets. - J. Fourier Anal. Appl., Vol. 3, No. 5, pp. 597-615.

Lyubarskii Y. and Spitkovsky I. (1996): Sampling and interpolation for a lacunary spectrum. — Proc. Royal. Soc. Edinburgh, Vol. 126 A, No. 1, pp. 77-87.

Moran W. and Avdonin S. (1999): Sampling of multi-band signals. - Proc. 4-th Int. Congress Industrial and Applied Mathematics, Edinburgh, Scotland, pp. 163-174.

Peterson K. (1983): Ergodic Theory. — Cambridge: Cambridge University Press.

Russell D. (1978): Controllability and stabilizability theory for linear partial differential equations. - SIAM Review, Vol. 20, No. 4, pp. 639-739.

Seip K. (1995): A simple construction of exponential bases in $L^{2}$ of the union of several intervals. — Proc. Edinburgh Math. Soc., Vol. 38, No. 1, pp. 171-177.

Spitkovsky I. (2006): Personal communication.

\section{Appendices}

\section{A. Proof of Theorem 2}

We single out several terms of the sum (9) that have smallest powers of $\mu$. The sum of those terms forms the operator $U$. We choose the number of terms so that later it will be possible to prove the invertibility of $U$. In the course of this proof, we shall show that this number of terms does not need to be greater than four.

1. We are looking for $r, k, q$ with the smallest possible $k$ such that $t-w(r, k, q) \in\left[0, \alpha_{1}\right]$ for some $t \in\left[\alpha^{\prime}, \alpha^{\prime}+\alpha_{1}\right]$.

2. Suppose that $\alpha^{\prime}-w(r, 0,0) \in\left[0, \alpha_{1}\right]$ or $\alpha^{\prime}+\alpha_{1}-$ $w(r, 0,0) \in\left[0, \alpha_{1}\right]$ for some $r$.

2.1. Let $\alpha^{\prime}-w(r, 0,0) \in\left[0, \alpha_{1}\right]$. Then $\alpha^{\prime}+\alpha_{1}-$ $w(r, 1,0)=\alpha^{\prime}-w(r, 0,0) \in\left[0, \alpha_{1}\right]$. Also, there may or may not be $\alpha^{\prime}+\alpha_{1}-w(r, 1,1) \in\left[0, \alpha_{1}\right]$.
2.1.1. If $\alpha^{\prime}+\alpha_{1}-w(r, 1,1) \in\left[0, \alpha_{1}\right]$, then

$$
\begin{aligned}
(U f)(t)= & \chi_{\left[\alpha^{\prime}, \alpha^{\prime}+\alpha_{1}\right]}(t)[A(r, 0,0) f(t-w(r, 0,0)) \\
& +A(r, 1,0) f(t-w(r, 1,0)) \\
& +A(r, 1,1) f(t-w(r, 1,1))] .
\end{aligned}
$$

2.1.2. If $\alpha^{\prime}+\alpha_{1}-w(r, 1,1) \notin\left[0, \alpha_{1}\right]$, then

$$
\begin{aligned}
(U f)(t) & =\chi_{\left[\alpha^{\prime}, \alpha^{\prime}+\alpha_{1}\right]}(t)[A(r, 0,0) f(t-w(r, 0,0)) \\
& +A(r, 1,0) f(t-w(r, 1,0))] .
\end{aligned}
$$

2.2. Let $\alpha^{\prime}+\alpha_{1}-w(r, 0,0) \in\left[0, \alpha_{1}\right)$. Since $\alpha^{\prime}>0$, we have $r>0$. Then $\alpha^{\prime}-w(r-1,1,1)=\alpha^{\prime}+\alpha_{1}-$ $w(r, 0,0) \in\left[0, \alpha_{1}\right)$. Also, $\alpha^{\prime}-w(r-1,1,0) \in\left[0, \alpha_{1}\right]$ may hold.

2.2.1. If $\alpha^{\prime}-w(r-1,1,0) \in\left[0, \alpha_{1}\right]$, then

$$
\begin{aligned}
(U f)(t)= & \chi_{\left[\alpha^{\prime}, \alpha^{\prime}+\alpha_{1}\right]}(t)[A(r, 0,0) f(t-w(r, 0,0)) \\
& +A(r-1,1,0) f(t-w(r-1,1,0)) \\
& +A(r-1,1,1) f(t-w(r-1,1,1))] \cdot(37)
\end{aligned}
$$

2.2.2. If $\alpha^{\prime}-w(r-1,1,0) \notin\left[0, \alpha_{1}\right]$ then

$$
\begin{aligned}
(U f)(t)= & \chi_{\left[\alpha^{\prime}, \alpha^{\prime}+\alpha_{1}\right]}(t)[A(r, 0,0) f(t-w(r, 0,0)) \\
& +A(r-1,1,1) f(t-w(r-1,1,1))] .
\end{aligned}
$$

3. Now we can assume that $t-w(r, 0,0) \notin\left[0, \alpha_{1}\right]$ for any $t \in\left[\alpha^{\prime}, \alpha^{\prime}+\alpha_{1}\right]$ and $r \geq 0$.

Observe that

$$
w(r, k, q)=w(r+q, k-2 q, 0) \quad \text { for } k \geq 2 q
$$

and

$w(r, k, q)=w(r+k-q, 2 q-k, 2 q-k)$ for $2 q \geq k$.

Therefore, $(r, k, q)$ with minimal $k$ such that $t-$ $w(r, k, q) \in\left[0, \alpha_{1}\right]$ for some $t \in\left[\alpha^{\prime}, \alpha^{\prime}+\alpha_{1}\right]$ will have the form of $(r, k, 0)$ or $(r, k, k)$, where $k \geq 1$.

4. Let us find $r_{1}$ and $k_{1}$ such that $\alpha^{\prime}-w\left(r_{1}, k_{1}, 0\right) \in$ $\left[0, \alpha_{1}\right]$ and $k_{1}$ is the smallest possible.

The answer is

$$
r_{1}=\left\lfloor\frac{\alpha^{\prime}}{\alpha}\right\rfloor, \quad k_{1}=\left\lfloor\frac{\alpha^{\prime}-r_{1} \alpha}{\alpha_{1}}\right\rfloor,
$$

where $\lfloor x\rfloor$ denotes the integer part of $x$. Also, $\alpha^{\prime}+\alpha_{1}-$ $w\left(r_{1}, k_{1}+1,0\right) \in\left[0, \alpha_{1}\right]$. 
Note that $k_{1}+1$ is the smallest $k_{2}$ such that $\alpha^{\prime}+$ $\alpha_{1}-w\left(r_{2}, k_{2}, 0\right) \in\left[0, \alpha_{1}\right]$. If there is $k_{2}<k_{1}+1$ with $\alpha^{\prime}+\alpha_{1}-w\left(r_{2}, k_{2}, 0\right) \in\left[0, \alpha_{1}\right]$, then $\alpha^{\prime}+w\left(r_{2}, k_{2}-\right.$ $1,0) \in\left[0, \alpha_{1}\right]$ and $k_{2}-1<k_{1}$ which is a contradiction.

5. Let us find $r_{3}, k_{3}, r_{4}, k_{4}$ such that $\alpha^{\prime}+\alpha_{1}-$ $w\left(r_{3}, k_{3}, k_{3}\right) \in\left[0, \alpha_{1}\right]$ and $\alpha^{\prime}-w\left(r_{4}, k_{4}, k_{4}\right) \in\left[0, \alpha_{1}\right]$ with the smallest $k_{3}, k_{4}$. It is easy to see that these conditions are satisfied by $k_{3}=\left\lfloor\frac{\left(r_{1}+1\right) \alpha-\alpha^{\prime}}{\alpha_{1}}\right\rfloor, r_{3}=r_{1}+1-k_{3}$, $k_{4}=k_{3}+1, r_{4}=r_{3}-1$.

6.1. Let $r_{3}<0$ or $k_{3}>k_{1}+1$. Then

$(U f)(t)$

$$
\begin{aligned}
= & \chi_{\left[\alpha^{\prime}, \alpha^{\prime}+\alpha_{1}\right]}(t)\left[A\left(r_{1}, k_{1}, 0\right) f\left(t-w\left(r_{1}, k_{1}, 0\right)\right)\right. \\
& \left.+A\left(r_{1}, k_{1}+1,0\right) f\left(t-w\left(r_{1}, k_{1}+1,0\right)\right)\right] .
\end{aligned}
$$

6.2. Let $r_{3}=0, k_{3} \leq k_{1}+1$. We have

$(U f)(t)$

$$
\begin{aligned}
= & \chi_{\left[\alpha^{\prime}, \alpha^{\prime}+\alpha_{1}\right]}(t)\left[A\left(r_{1}, k_{1}, 0\right) f\left(t-w\left(r_{1}, k_{1}, 0\right)\right)\right. \\
& \left.+A\left(r_{1}, k_{1}+1,0\right) f\left(t-w\left(r_{1}, k_{1}+1,0\right)\right)\right) \\
& \left.+A\left(0, k_{3}, k_{3}\right) f\left(t-w\left(0, k_{3}, k_{3}\right)\right)\right] .
\end{aligned}
$$

6.3. Let $r_{3}>0$ and $k_{3}=k_{1}+1$. Then $r_{3}=r_{1}-k_{1}$. We get

$$
\begin{aligned}
& (U f)(t) \\
& =\chi_{\left[\alpha^{\prime}, \alpha^{\prime}+\alpha_{1}\right]}(t)\left[A\left(r_{1}, k_{1}, 0\right) f\left(t-w\left(r_{1}, k_{1}, 0\right)\right)\right. \\
& \quad+A\left(r_{1}, k_{1}+1,0\right) f\left(t-w\left(r_{1}, k_{1}+1,0\right)\right) \\
& \quad+A\left(r_{1}-k_{1}, k_{1}+1, k_{1}+1\right) \\
& \left.\quad \times f\left(t-w\left(r_{1}-k_{1}, k_{1}+1, k_{1}+1\right)\right)\right] .
\end{aligned}
$$

6.4. Let $r_{3}>0$ and $k_{3}=k_{1}$. Then

$$
\begin{aligned}
(U f) & (t) \\
= & \chi_{\left[\alpha^{\prime}, \alpha^{\prime}+\alpha_{1}\right]}(t)\left[A\left(r_{1}, k_{1}, 0\right) f\left(t-w\left(r_{1}, k_{1}, 0\right)\right)\right. \\
& +A\left(r_{1}, k_{1}+1,0\right) f\left(t-w\left(r_{1}, k_{1}+1,0\right)\right) \\
& +A\left(r_{1}-k_{1}+1, k_{1}, k_{1}\right) \\
& \times f\left(t-w\left(r_{1}-k_{1}+1, k_{1}, k_{1}\right)\right) \\
& +A\left(r_{1}-k_{1}, k_{1}+1, k_{1}+1\right) \\
& \left.\times f\left(t-w\left(r_{1}-k_{1}, k_{1}+1, k_{1}+1\right)\right)\right] .
\end{aligned}
$$

6.5. Let $r_{3}>0$ and $k_{3}=k_{1}-1$. We have

$$
\begin{aligned}
& (U f)(t) \\
& =\chi_{\left[\alpha^{\prime}, \alpha^{\prime}+\alpha_{1}\right]}(t)\left[A\left(r_{1}, k_{1}, 0\right) f\left(t-w\left(r_{1}, k_{1}, 0\right)\right)\right. \\
& \quad+A\left(r_{1}-k_{1}+2, k_{1}-1, k_{1}-1\right) \\
& \quad \times f\left(t-w\left(r_{1}-k_{1}+2, k_{1}-1, k_{1}-1\right)\right) \\
& \quad+A\left(r_{1}-k_{1}+1, k_{1}, k_{1}\right) \\
& \left.\quad \times f\left(t-w\left(r_{1}-k_{1}+1, k_{1}, k_{1}\right)\right)\right] .
\end{aligned}
$$

6.6. Suppose that $r_{3}>0$ and $k_{3}<k_{1}-1$. We get

$$
\begin{aligned}
(U f)(t) & \\
= & \chi_{\left[\alpha^{\prime}, \alpha^{\prime}+\alpha_{1}\right]}(t)\left[A\left(r_{3}, k_{3}, k_{3}\right) f\left(t-w\left(r_{3}, k_{3}, k_{3}\right)\right)\right. \\
& +A\left(r_{3}-1, k_{3}+1, k_{3}+1\right) \\
& \left.\times f\left(t-w\left(r_{3}-1, k_{3}+1, k_{3}+1\right)\right)\right] .
\end{aligned}
$$

We derived all possible formulae for $U$ for various relative positions of the intervals $I_{1}$ and $I_{2}$ (see (8)). Note that $U$ may contain two (formulae (36), (38), (39), (44)), three (formulae (35), (37), (40), (41), (43)), or four (formula (42)) terms.

\section{B. Proof of Lemma 1}

First we show that $\|U f\| \geq|\mu|^{k+1} B\|f\|$ for any $f \in$ $L^{2}\left[0, \alpha_{1}\right]$, where $B$ is a positive constant. To prove this, we need the formulae for the operator $U$ from the proof of Theorem 2 given in Appendix A. We consider separately cases when $U$ consists of 2, 3 and 4 terms.

In (36), (38), (39) and (44), the operator $U$ has two terms:

$$
\begin{aligned}
(U f)(t)= & \chi_{\left[\alpha^{\prime}, \alpha^{\prime}+\alpha_{1}\right]}(t)\left[\mu^{k} A_{1} f(t-w)\right. \\
& \left.+\mu^{k+1} A_{2} f\left(t-w \pm \alpha_{1}\right)\right] .
\end{aligned}
$$

Here $A_{1}, A_{2}, w$ do not depend on $\mu$, and $A_{1} \neq 0, A_{2} \neq 0$. Observe that, since $f$ is defined on $\left[0, \alpha_{1}\right]$, the two terms are never non-zero on the same part of the interval $\left[0, \alpha_{1}\right]$, because the distance between $t-w$ and $t-w \pm \alpha_{1}$ is $\alpha_{1}$. Then

$$
\begin{aligned}
\|(U f)(t)\| & \geq \min \left(\left|A_{1}\right|,\left|\mu A_{2}\right|\right)|\mu|^{k}\|f\| \\
& \geq|\mu|^{k+1}\left|A_{2}\right|\|f\|
\end{aligned}
$$

for sufficiently small $\mu$.

Let us consider the cases when the operator $U$ has three terms. In (35), (37), (41) and (43), the operator $U$ has the form

$$
\begin{aligned}
& (U f)(t) \\
& =\chi_{\left[\alpha^{\prime}, \alpha^{\prime}+\alpha_{1}\right]}(t)\left[\mu^{k} A_{1} f\left(t-w_{1}\right)\right. \\
& \left.\quad+\mu^{k+1} A_{2} f\left(t-w_{1} \pm \alpha_{1}\right)+\mu^{k+1} A_{3} f\left(t-w_{2}\right)\right] .
\end{aligned}
$$


For these cases,

$$
\begin{aligned}
\|(U f)(t)\| & \geq \min \left(\left|A_{1}\right|,\left|\mu A_{2}\right|,\left|A_{1}+\mu A_{3}\right|,\right. \\
\left.\left|\mu\left(A_{2}+A_{3}\right)\right|\right)|\mu|^{k}\|f\| & \geq|\mu|^{k+1} \min \left(\left|A_{2}+A_{3}\right|,\left|A_{2}\right|\right)\|f\|
\end{aligned}
$$

for sufficiently small $\mu$. Using the exact formulae for the coefficients $A_{i}$, one can check that $A_{2}+A_{3} \neq 0$.

In (40), the operator $U$ is

$(U f)(t)$

$$
\begin{aligned}
= & \chi_{\left[\alpha^{\prime}, \alpha^{\prime}+\alpha_{1}\right]}(t)\left[\mu^{k} A_{1} f\left(t-w_{1}\right)\right. \\
& \left.+\mu^{k+1} A_{2} f\left(t-w_{1} \pm \alpha_{1}\right)+\mu^{k_{3}} A_{3} f\left(t-w_{2}\right)\right],
\end{aligned}
$$

$k_{3} \leq k+1$. Then, for sufficiently small $\mu$ and a positive constant $D$, we have

$$
\begin{aligned}
\|(U f)(t)\| & \geq \min \left(\left|A_{1}\right|,\left|\mu A_{2}\right|,\left|A_{1}+\mu^{k_{3}-k} A_{3}\right|,\right. \\
\left.\left.\mid \mu A_{2}+\mu^{k_{3}-k} A_{3}\right) \mid\right)|\mu|^{k}\|f\| & \\
& \geq|\mu|^{k+1} \min \left(\left|A_{2}+\mu^{k_{3}-k-1} A_{3}\right|,\right. \\
\left.\left|A_{2}\right|\right)\|f\| & \geq D|\mu|^{k+1}\|f\| .
\end{aligned}
$$

Now we consider the last case of four terms (see (42)):

$$
\begin{aligned}
& (U f)(t) \\
& =\chi_{\left[\alpha^{\prime}, \alpha^{\prime}+\alpha_{1}\right]}(t) \\
& \times\left[\mu^{k} A_{1} f\left(t-w_{1}\right)+\mu^{k+1} A_{2} f\left(t-w_{1}-\alpha_{1}\right)\right. \\
& \left.+\mu^{k} A_{3} f\left(t-w_{2}\right)+\mu^{k+1} A_{4} f\left(t-w_{2}+\alpha_{1}\right)\right] \text {. }
\end{aligned}
$$

Then

$$
\begin{array}{r}
\|(U f)(t)\| \geq \min \left(\left|A_{1}+A_{3}\right|,\left|A_{1}+\mu A_{4}\right|,\right. \\
\left.\left|A_{3}+\mu A_{2}\right|,\left|\mu\left(A_{2}+A_{4}\right)\right|\right) \\
|\mu|^{k}\|f\| \geq|\mu|^{k+1}\left|A_{2}+A_{4}\right|\|f\|
\end{array}
$$

for sufficiently small $\mu$.

Let us show that $A_{2}+A_{4} \neq 0$. From (42), we see that

$$
\begin{aligned}
& A_{2}=(-1)^{r+k+1} \frac{(r+k+1) !}{r !(k+1) !} \\
& A_{4}=(-1)^{r+1} \frac{(r+1) !}{(r-k) !(k+1) !}
\end{aligned}
$$

for some $r, k \geq 1$. Note that, for $k \geq 1$,

$$
\frac{(r+k+1) !}{r !(k+1) !}>\frac{(r+1) !}{(r-k) !(k+1) !}
$$

and hence $A_{2}+A_{4} \neq 0$.

We have shown that for any $f \in L^{2}\left(\left[0, \alpha_{1}\right]\right)$, we have that $\|U f\| \geq|\mu|^{k+1} B\|f\|$, where $B$ is a positive non-zero constant. Since we assumed that $U$ is invertible, for any $g \in L^{2}\left(\left[\alpha^{\prime}, \alpha^{\prime}+\alpha_{1}\right]\right)$, we have $\|g\| \geq|\mu|^{k+1} B\left\|U^{-1} g\right\|$. Consequently, for every $g \in L^{2}\left(\left[\alpha^{\prime}, \alpha^{\prime}+\alpha_{1}\right]\right)$, we have $\left\|U^{-1} g\right\| \leq|\mu|^{-(k+1)} \frac{1}{B}\|g\|$. Thus $\left\|U^{-1}\right\| \leq|\mu|^{-(k+1)} C$.

Received: 2 November 2006 Revised: 22 February 2007 